\title{
The dynamics of vibrational excitations on surfaces: CO on Ru(001)
}

Cite as: J. Chem. Phys. 115, 7725 (2001); https://doi.org/10.1063/1.1404986

Submitted: 22 May 2001 . Accepted: 31 July 2001 . Published Online: 12 October 2001

Mischa Bonn, Christian Hess, and Martin Wolf

\section{ARTICLES YOU MAY BE INTERESTED IN}

Desorption of CO from Ru(001) induced by near-infrared femtosecond laser pulses The Journal of Chemical Physics 112, 9888 (2000); https://doi.org/10.1063/1.481626

Frequency- and time-domain femtosecond vibrational sum frequency generation from CO adsorbed on Pt(111)

The Journal of Chemical Physics 121, 10174 (2004); https://doi.org/10.1063/1.1802291

Vibrational energy transfer of $\mathrm{CO} / \mathrm{Cu}(100)$ : Nonadiabatic vibration/electron coupling

The Journal of Chemical Physics 96, 3950 (1992); https://doi.org/10.1063/1.461897
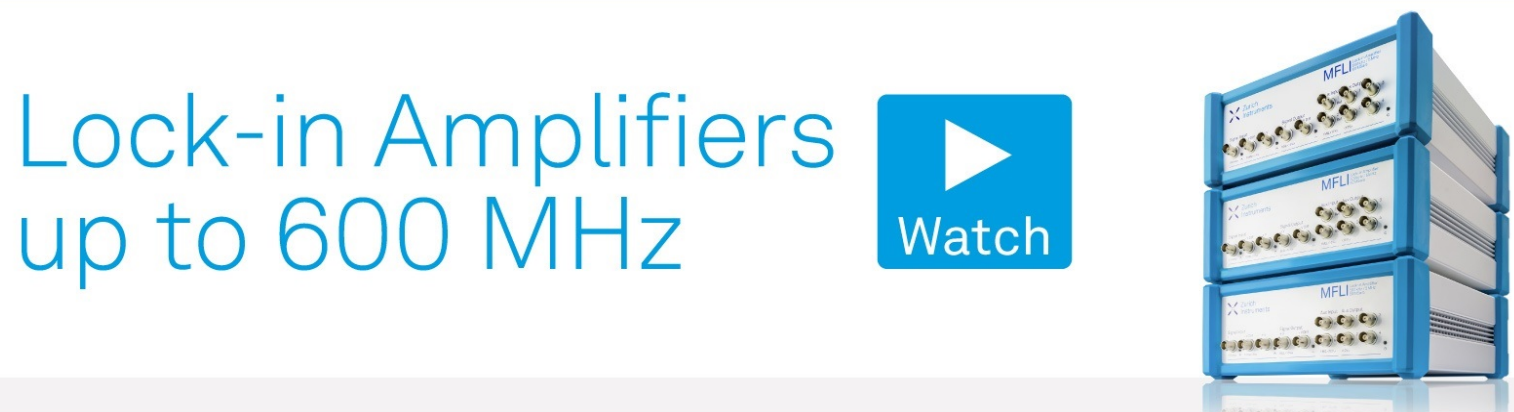

J. Chem. Phys. 115, 7725 (2001); https://doi.org/10.1063/1.1404986 


\title{
The dynamics of vibrational excitations on surfaces: CO on Ru(001)
}

\author{
Mischa Bonn ${ }^{\text {a) }}$ \\ Leiden Institute of Chemistry, P.O. Box 9502, 2300 RA Leiden, The Netherlands \\ Christian Hess \\ Fritz-Haber-Institut der Max-Planck-Gesellschaft, Faradayweg 4-6, 14195 Berlin, Germany \\ Martin Wolf \\ Fritz-Haber-Institut der Max-Planck-Gesellschaft, Faradayweg 4-6, 14195 Berlin, Germany \\ and Freie Universität Berlin, Institut für Experimentalphysik, Arnimalle 14, 14195 Berlin, Germany
}

(Received 22 May 2001; accepted 31 July 2001)

\begin{abstract}
We present an experimental and theoretical study of vibrational excitation of the $\mathrm{C}-\mathrm{O}$ stretch vibration of carbon monoxide adsorbed on a ruthenium $\mathrm{Ru}(001)$ surface with ultrashort femtosecond infrared laser pulses. After broadband excitation leading to transfer of a significant fraction of the $\mathrm{CO}$ molecules to their first $(\sim 15 \%)$ and second $(\sim 5 \%)$ vibrationally excited states, we observe a competition between vibrational energy relaxation and energy delocalization through dipole-dipole coupling. We reproduce the observed excited state spectra by solving the three-level Bloch equations and accounting for intermolecular vibrational energy transfer on a picosecond time scale. The rate of vibrational energy transfer, and its coverage-dependence, can be described by a Förster energy transfer mechanism. We discuss possibilities to optimize the degree of localized vibrational excitation of a specific bond of molecules at surfaces through chirped pulse IR excitation. (C) 2001 American Institute of Physics. [DOI: 10.1063/1.1404986]
\end{abstract}

\section{INTRODUCTION}

It is one of the goals in physical chemistry to achieve control over chemical reactions on a molecular level. Since the vibrational state of a molecule plays a key role in determining its reactivity, excitation of a specific vibrational mode is an important aspect of state-selective chemistry. Control over the outcome of a chemical reaction by mode-specific vibrational excitation has been demonstrated in detail in the gas phase. For instance, the outcome of the reaction between a hydrogen atom and (deuterated) water to give $\mathrm{H}_{2}(\mathrm{HD})$ and $\mathrm{OH}(\mathrm{OD})$, can be controlled to a large extent by bondspecifically vibrationally exciting either the $\mathrm{O}-\mathrm{H}$ or the $\mathrm{O}-\mathrm{D}$ stretch vibration of the reactant water; ${ }^{1-3}$ the bond that is vibrationally excited preferentially reacts. It is clear that a higher degree of vibrational excitation, viz. excitation to high-lying vibrational states, will increase the selectivity. Due to the anharmonicity of the vibrational ladder, broadband infrared laser sources are ideally suited for the "vibrational ladder-climbing" process. ${ }^{4,5}$ In addition, these short laser pulses have the advantage that the energy is inserted into the system faster than vibrational relaxation (IVR) and energy redistribution can occur, so that the energy remains localized in the relevant coordinate long enough for the subsequent chemical transition to occur. This is particularly important for larger molecules and, as will be demonstrated below, for molecules on surfaces. Indeed, it has been demonstrated for gas-phase NO that ultrashort infrared pulses can be used to transfer population up to the highest level of the $\mathrm{N}-\mathrm{O}$ stretch vibration within reach of the IR bandwidth, i.e., up to $v=5 .^{6}$ Equally high levels of vibrational excitation

\footnotetext{
a) Author to whom correspondence should be addressed. Electronic mail: m.bonn@chem.leidenuniv.nl
}

have been obtained after resonant broadband excitation the $\mathrm{C}-\mathrm{O}$ stretching vibration of $\mathrm{W}(\mathrm{CO})_{6}$ in solution. ${ }^{7}$ Moreover, frequency-chirped laser pulses can considerably enhance the efficiency of the population transfer, both for gas-phase NO (Ref. 8) and for liquid phase $\mathrm{W}(\mathrm{CO})_{6} .{ }^{9}$ These chirped pulses have a time-dependent frequency, that follow the consecutive vibrational transitions in an anharmonic potential: first the molecule is offered the high frequency components within the bandwidth to climb the lower steps of the ladder, after which the low-frequency components can be used to reach higher-lying vibrational states.

For molecules on surfaces, steering reactions by means of infrared excitation has not yet developed to an equal degree of sophistication. Although on insulator surfaces very high degrees of vibrational excitation can be achieved through vibrational energy pooling, ${ }^{10}$ and adsorbates have been chemically activated through IR excitation, ${ }^{11}$ on metal surfaces two severe complications occur: first, due to efficient coupling with the electron continuum in the underlying substrate, efficient vibrational energy relaxation will occur by electron-hole pair excitation in the metal, typically on a picosecond time scale. ${ }^{12,13}$ This means that there will be competition between excitation and de-excitation, especially relevant for chirped pulses, which are inherently longer than bandwidth-limited pulses. Second, the proximity and preferential alignment of molecules on the surface may give rise to dipole-dipole coupling between adsorbates, effectively delocalizing the vibrational energy (for $\mathrm{CO}$ and $\mathrm{NO}$ on $\mathrm{Ru}$, see for instance Refs. 14, 15, and 16 respectively). The exchange of vibrational energy on semi-conductor surfaces has been observed among different $\mathrm{Si}-\mathrm{H}$ stretch vibrations for hydrogen adsorbed on a stepped silicon surface. ${ }^{17-19}$ However, mode-selective chemistry requires localization of vibrational 

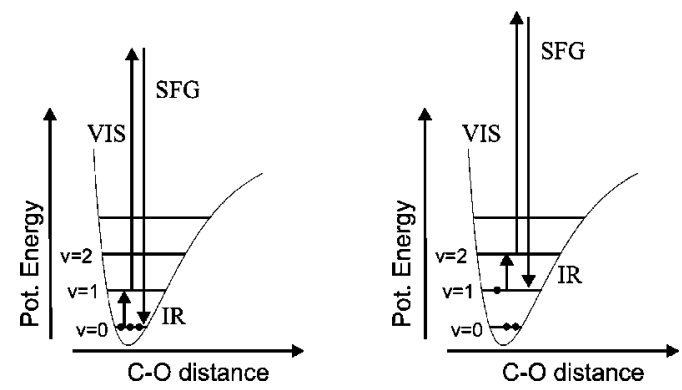

FIG. 1. Schematic representation of conventional sum-frequency generation (SFG) spectroscopy (left panel): An enhancement of the SFG intensity is observed when the infrared radiation is resonant with a vibrational transition. Right panel: For a sufficiently intense (broadband) infrared pulse, significant population transfer will occur to the first excited state $v=1$. Subsequently, SFG from the $v=1 \rightarrow 2$ transition can occur, which will be slightly red-shifted from the $v=0 \rightarrow 1$ due to the anharmonicity of the potential.

energy in a specific bond, as has been demonstrated for multi-vibrational excitation (and desorption) of $\mathrm{H} / \mathrm{Si}$ induced by inelastic tunneling in scanning tunneling microscopy ${ }^{20}$ but so far not for optical excitation. A deeper understanding of the dynamics of vibrational energy transfer on metal surfaces and controlled vibrational excitation is therefore desirable.

We present here an investigation into the dynamics of vibrational excitations on surfaces, in particular into the $\mathrm{C}-\mathrm{O}$ stretch vibration of carbon monoxide absorbed on a $\mathrm{Ru}(001)$ surface. We demonstrate that, after a vibrational excitation with ultrashort infrared pulses, there is a competition between localization on the one hand and quenching (vibrational relaxation) and delocalization ${ }^{14,15,21}$ on the other hand. The delocalization is a consequence of dipole-dipole coupling between the molecules on the surface. The degree of delocalization can be controlled by varying the surface coverage, and the delocalization dynamics can be described very well by a Förster model for vibrational energy transfer. Vibrational relaxation is quite efficient and calculations show that the adverse effect of vibrational relaxation on populating higher-lying states can be circumvented only to a limited extent by chirping the excitation pulses, in contrast to gasphase experiments.

We study these phenomena by means of sum-frequency generation spectroscopy (SFG) with intense, femtosecond infrared pulses. The basic scheme behind sum-frequency generation is illustrated in Fig. 1. SFG allows one to record the vibrational spectrum of molecules adsorbed on a surface in the submonolayer coverage regime. ${ }^{22}$ For the high infrared intensities used in the present study, a considerable fraction of the molecules can be vibrationally excited. This allows for the SFG from higher-lying vibrational states, as illustrated in the right panel of Fig. 1.

The behavior of the SFG signal as a function of coverage and infrared intensity contains important information about different aspects of dynamics of vibrations at surfaces, as will be demonstrated below. The experimental section is followed by a section regarding vibrational ladder climbing on surfaces, and the theoretical description of this phenomenon in terms of the Bloch equations. We will also discuss the possibility of achieving higher levels of excitation by chirp-

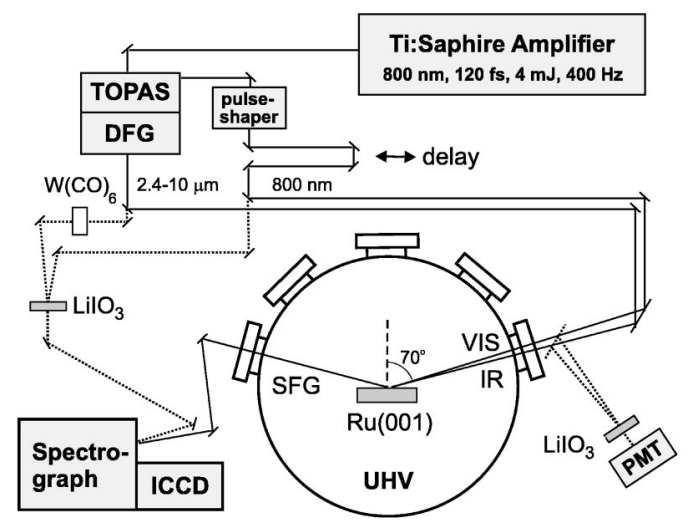

FIG. 2. Schematic overview of the experimental setup: Solid lines indicate the optical setup used for broadband-IR sum-frequency-generation (SFG) spectroscopy. Dashed lines refer to the $\mathrm{W}(\mathrm{CO})_{6}$ reference used for frequency calibration as well as for optimizing the spatial and temporal overlap between the IR and VIS-upconversion beam (see text).

ing the infrared pulse. In Sec. IV we present experimental data on vibrational energy transfer among molecules on surfaces. It is demonstrated that this can be described very accurately in terms of resonant Förster energy transfer. We conclude by presenting a brief summary and conclusions. In the Appendix we demonstrate some effects of saturation on the experimental results, the interpretation and the correct treatment of this artifact.

\section{EXPERIMENT}

The experiments were performed with a femtosecond laser system combined with an ultrahigh vacuum (UHV) chamber which is schematically displayed in Fig. 2. An optical parametric amplifier (TOPAS, Light Conversion) pumped by $4 \mathrm{~mJ}$ of an amplified Ti:Sapphire laser system (Titan II, Quantronix, $800 \mathrm{~nm}, 120 \mathrm{fs}, 400 \mathrm{~Hz}$ repetition rate) is used to generate tunable near-IR pulses (signal and idler, $\sim 1-2.5 \mu \mathrm{m})$ in BBO. Mid-IR pulses $(2-10 \mu \mathrm{m})$ are subsequently generated by difference frequency mixing (DFG) of signal and idler in $\mathrm{AgGaS}_{2}$ (Type I, thickness $1 \mathrm{~mm}$ ). At a wavelength of about $5 \mu \mathrm{m}$ femtosecond IR-pulses with energies up to $25 \mu \mathrm{J}$ and a bandwidth of typically $100-120$ $\mathrm{cm}^{-1}$ (FWHM) are obtained. The residual $800 \mathrm{~nm}$ light after the parametric generation is separated from the signal and idler by a dielectric beamsplitter and spectrally narrowed to a bandwidth of $5-8 \mathrm{~cm}^{-1}$ using a pulse shaper (2000 grooves/mm grating, variable slit combination). The spectral narrowing results in a temporal spread for the $800 \mathrm{~nm}$ (VIS) upconversion pulse between 1 and $4 \mathrm{ps,}$, as verified by crosscorrelation measurements. After passing a variable delay the VIS upconversion pulses are spatially and temporally overlapped with the IR pulses in a $\mathrm{LiIO}_{3}$ crystal outside the UHV chamber at a position equivalent to the sample (Fig. 2, dashed lines). For the SFG experiments the IR and VIS pulses are focused into the UHV chamber under an angle of $70^{\circ}$ with respect to the surface normal. Typical pulse energies are $4 \mu \mathrm{J}$ for the VIS and up to $11 \mu \mathrm{J}$ for the IR pulses 


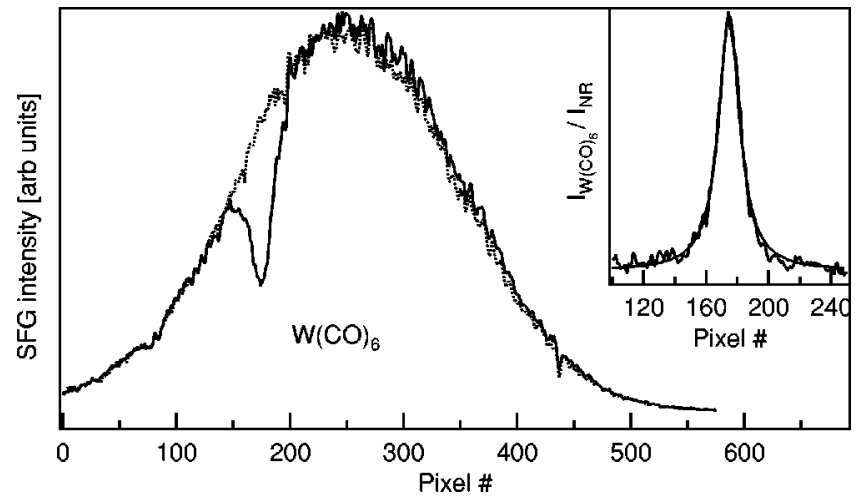

FIG. 3. IR+VIS-sum-frequency-generation signal with (solid line) and without (dotted line) a $1 \mathrm{~mm}$ cell containing a $10^{-3} \mathrm{M}$ solution of $\mathrm{W}(\mathrm{CO})_{6}$ in $\mathrm{CCl}_{4}$ in the infrared beam path. The SFG is generated in an appropriately cut, phase-matched $\mathrm{LiIO}_{3}$ crystal. The ratio of the two signals shown in the inset reveals the sharp resonance associated with the CO-stretch of $\mathrm{W}(\mathrm{CO})_{6}$, used for calibration purposes.

at the sample (beam waist at focus, $0.3 \mathrm{~mm}$ FWHM). The center frequency of the IR is resonant with the $\mathrm{C}-\mathrm{O}$ stretch vibration.

SFG is a second-order nonlinear optical process in which two incident waves at $\omega_{\mathrm{VIS}}$ and $\omega_{\mathrm{IR}}$ generate an output at $\omega_{\mathrm{SFG}}=\omega_{\mathrm{VIS}}+\omega_{\mathrm{IR}}$, where energy and momentum must be conserved. ${ }^{22}$ When using broadband-IR pulses, resonant enhancement will only occur for frequencies $\omega_{\text {IR }}$ within the bandwidth which are resonant with the vibrational transition. Thus, vibrational spectra can be obtained without scanning the IR frequency. ${ }^{23-25}$ The SFG beam is focused into a spectrograph and dispersed across an intensified $\mathrm{CCD}$ detector.

As a reference for frequency calibration a $1 \mathrm{~mm} \mathrm{CaF}_{2}$ cell containing a $10^{-3} \mathrm{M}$ solution of $\mathrm{W}(\mathrm{CO})_{6}$ in $\mathrm{CCl}_{4}$ is used in the IR beam path (Fig. 3, dashed lines). The transmitted IR spectrum is upconverted in a $\mathrm{LiIO}_{3}$ crystal with the narrowband VIS upconversion pulse and send to the spectrograph. The resulting SFG spectrum is displayed in Fig. 3 in comparison with the spectrum of the pure solvent. The Lorentzian absorption [literature value: $1980 \mathrm{~cm}^{-1}$ (Ref. 26)] allows for an absolute calibration within $1 \mathrm{~cm}^{-1}$ the peak for the reference is set at this frequency. The dispersion per pixel on the CCD camera can simply be obtained by scanning the spectrometer.

The $\mathrm{Ru}(001)$ sample is mounted in an UHV chamber (base pressure $1 \times 10^{-10} \mathrm{mbar}$ ) and can be cooled to $95 \mathrm{~K}$. An extensive description of the complete experimental setup and surface cleaning procedures can be found in Ref. 27. CO exposure was performed via a pinhole doser or a variable leak valve. The $\mathrm{CO}$ coverage is determined by comparing the integrated desorption yield with thermal desorption spectra obtained after saturating the Ru surface with $\mathrm{CO}[0.68 \mathrm{ML}$ (Ref. 28)]. For all coverages reported here, $\mathrm{CO}$ is adsorbed on atop $\mathrm{Ru}$ sites. ${ }^{28}$ Transient surface temperature increases due to nonresonant laser heating by the VIS upconversion pulse are below $30 \mathrm{~K}$ and are therefore unimportant in the interpretation of the data.
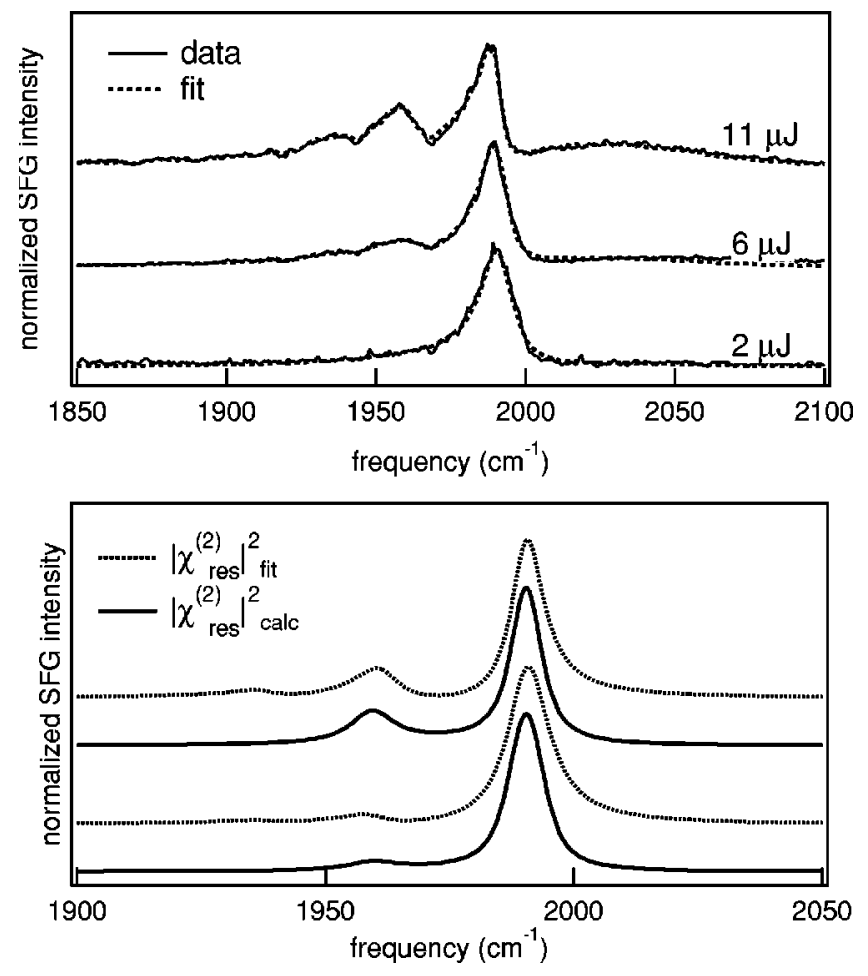

FIG. 4. Upper panel: Normalized SFG spectra of the CO-stretch of CO on $\mathrm{Ru}(001)$ as a function of IR energy at $95 \mathrm{~K}$ (gray lines) together with the least-squares fits to the data (dotted lines). Starting with an IR energy of 11 $\mu \mathrm{J}$ at a coverage of about $0.007 \mathrm{ML}$ a series of spectra was recorded as a function of decreasing IR energy while dosing $\mathrm{CO}$ via the background. At 11 $\mu \mathrm{J}$ the fundamental and $v=1 \rightarrow 2$ hot band transition are clearly visible. The third resonance is partly due to the $v=2 \rightarrow 3$ hot band. Lower panel: Resonant part of the fit depicted in the upper panel for the 6 (lower trace) and 11 $\mu \mathbf{J}$ (upper trace) experiments (dotted lines) and the results of the calculation (solid line) described in the text.

\section{VIBRATIONAL LADDER CLIMBING}

The upper panel of Fig. 4 depicts SFG spectra as a function of infrared intensity at a surface coverage of approximately 0.01 monolayers and a temperature of $95 \mathrm{~K}$. The coverage was kept sufficiently low to avoid effects of intermolecular coupling (see below). At low IR energies, a Lorentzian resonance is observed associated with the transition from the ground $(v=0)$ to the first excited $(v=1)$ state. With increasing energy the fundamental transition $(v=0$ $\rightarrow 1)$ is saturated, and significant population is transferred to the $(v=1)$ state. An additional interaction with the broadband infrared field gives rise to a polarization at the $(v=1$ $\rightarrow 2$ ) transition, from which SFG is generated at a slightly lower frequency due to the anharmonicity of the vibrational potential. This effect exhibits a strong nonlinear dependence on the infrared energy [as it is a fourth-order $\left(\chi^{(4)}\right)$ nonlinear optical process, see below], which is obvious from Fig. 4: With increasing IR power the $v=1 \rightarrow 2$ hot band of the COstretch vibration becomes clearly visible at $1961.4 \pm 0.3$ $\mathrm{cm}^{-1}$, in addition to the fundamental transition at 1990.4 $\pm 0.1 \mathrm{~cm}^{-1}$ which is also observed at lower IR energies. From these values the anharmonicity constant is determined to be $13.6 \pm 0.36 \mathrm{~cm}^{-1} .{ }^{29}$ The third resonance around 1938 $\mathrm{cm}^{-1}$ arises from two contributions: The $v=2 \rightarrow 3$ hot-band and the fundamental transition of the natural abundant 
${ }^{13} \mathrm{C}^{16} \mathrm{O}$ in ${ }^{12} \mathrm{C}^{16} \mathrm{O}$ gas. The SFG spectra $I_{\mathrm{SFG}}(\omega)$ can be reproduced very well by an expression for the second-order nonlinear susceptibility consisting of a nonresonant term $\chi_{\mathrm{NR}}^{(2)}$ arising from the surface region of the metal and a resonant term $\chi_{\mathrm{R}}^{(2)}$ associated with the vibrational transition, ${ }^{29,30}$

$$
\begin{aligned}
& I_{\mathrm{SFG}}(\omega) \propto\left|\chi^{(2)}\right|^{2} ; \quad \chi^{(2)}=\chi_{\mathrm{NR}}^{(2)}+\chi_{\mathrm{R}}^{(2)} ; \\
& \chi_{\mathrm{NR}}^{(2)}=A_{0} e^{\mathrm{i} \phi} ; \quad \chi_{R}^{(2)}=\sum_{n} \frac{A_{n}}{\omega_{\mathrm{IR}}-\omega_{n}+\mathrm{i} \Gamma_{n}},
\end{aligned}
$$

where the vibrational resonances are described by their resonance frequencies $\omega_{n}$, linewidths $2 \Gamma_{n}$ and amplitudes $A_{n}$, so that the $\chi^{(4)}$ contribution is incorporated in $\chi^{(2)} . A_{0}$ is the amplitude of the nonresonant susceptibility and $\phi$ its phase relative to the vibrational resonance. The dotted lines in the upper panel of Fig. 4 depict the fit to the experimental data using this procedure. The dotted lines in the lower panel show the resonant contribution to the nonlinear susceptibility as obtained from the fit.

The resonant contributions to the transient spectra can be calculated from the time-dependent polarization of the ground $(v=0 \rightarrow 1)$ and excited state $(v=1 \rightarrow 2)$ transitions that are related to the off-diagonal elements of the density matrix, as $P(\omega)=e^{i \omega_{\mathrm{VIS}} t} \times \operatorname{Tr}(\bar{\rho} \bar{\mu})$. The off-diagonal density matrix elements $\rho_{i j}$ oscillate at $\omega_{i j} \approx \omega_{\mathrm{IR}}$ for near-resonant excitation. In the rotating frame, the equations of motion for the different matrix elements of $\bar{\rho}$ read: $:^{31-33}$

$$
\begin{aligned}
\dot{\tilde{\rho}}_{00}= & -i V_{01} \tilde{\rho}_{10}+\text { c.c. }+\tilde{\rho}_{11} / T_{1}^{10}, \\
\dot{\tilde{\rho}}_{01}= & i V_{01}\left(\tilde{\rho}_{00}-\tilde{\rho}_{11}\right)+i V_{21} \tilde{\rho}_{02} \\
& -\tilde{\rho}_{01}\left[1 /\left(2 T_{1}^{10}\right)+1 / T_{2}^{01}+i \Omega_{01}\right], \\
\dot{\tilde{\rho}}_{11}= & -i V_{12} \tilde{\rho}_{21}+\text { c.c. }+i V_{01} \tilde{\rho}_{10}+\text { c.c. }-\tilde{\rho}_{11} / T_{1}^{10} \\
& +\tilde{\rho}_{22} / T_{1}^{21}, \\
\dot{\tilde{\rho}}_{12}= & i V_{12}\left(\tilde{\rho}_{11}-\tilde{\rho}_{22}\right)-i V_{10} \tilde{\rho}_{02}-\tilde{\rho}_{12}\left[1 /\left(2 T_{1}^{10}\right)\right. \\
& \left.+1 /\left(2 T_{1}^{21}\right)+1 / T_{2}^{12}+i \Omega_{12}\right], \\
\dot{\tilde{\rho}}_{02}= & -i\left(V_{01} \tilde{\rho}_{12}-V_{12} \tilde{\rho}_{01}\right)-\tilde{\rho}_{02}\left[1 /\left(2 T_{1}^{21}\right)\right. \\
& \left.+1 / T_{2}^{02}+i \Omega_{02}\right] .
\end{aligned}
$$

The above equations are the independent equations for the nine elements of the density matrix elements for the threelevel system. In addition we have:

$$
\begin{aligned}
& \tilde{\rho}_{22}=1-\tilde{\rho}_{00}-\tilde{\rho}_{11}, \\
& \tilde{\rho}_{10}=\tilde{\rho}_{01}^{*}, \\
& \tilde{\rho}_{21}=\tilde{\rho}_{12}^{*}, \\
& \tilde{\rho}_{20}=\tilde{\rho}_{20}^{*} .
\end{aligned}
$$

In these equations, $\tilde{\rho}_{i j}=\rho_{i j} e^{i\left(\Omega_{i j}\right) t}, \quad V_{i j}=-\mu_{i j} /(2 \hbar)$ $\mathcal{E}(t), T_{1}^{a b}$ and $T_{2}^{a b}$ denote the population and pure dephasing lifetimes of transition $a \rightarrow b$, respectively, and the detuning
$\Omega_{i j}=\omega_{i j}-\omega_{\mathrm{IR}} \cdot \mu_{i j}$ is the transition dipole moment of the transition from $i$ to $j$ and $\mathcal{E}(t)$ the envelope of the incident IR field.

The set of Eqs. (1)-(5) is solved using a fourth-order Runge-Kutta scheme, after decomposing Eqs. (3)-(5) into their real and imaginary parts, resulting in eight coupled differential equations. In these calculations, we set $T_{1}^{10}=T_{1}^{21}$, since the available phase-space for electron-hole pair creation in the relaxation processes are very similar, because almost the same amount of energy is involved in the two relaxation processes. We neglect population decay from the second excited state directly to the ground state, since the matrix element associated with this forbidden transition is expected to be very small. Furthermore, we set $\mu_{12}=2 \mu_{01}$, as for an harmonic oscillator, ${ }^{34}$ with $\mu_{01}=2.1 \times 10^{-30}$ $\mathrm{C} \mathrm{m} .{ }^{35}$ The $T_{2}$ 's are obtained from linewidth measurements of the fundamental $\left[T_{2}^{01}\right.$ corresponding to a linewidth of 3 $\mathrm{cm}^{-1}$ (Ref. 15)], hot band transitions $\left[T_{2}^{12}\right.$ corresponding to a linewidth of $15 \mathrm{~cm}^{-1}$ (Ref. 29)], and overtone [ $T_{2}^{02}$ corresponding to a linewidth of $6 \mathrm{~cm}^{-1}$ (Ref. 36)] reported previously. Finally, the observed resonant part of the SFG spectrum can be calculated from $I_{\text {res }}(\omega) \propto\left|\chi_{\text {res }}^{(2)}\right|^{2} \propto|P(\omega)|^{2}$, with the polarization $P(\omega)=e^{i \omega_{\mathrm{VIS}} t} \operatorname{Tr}(\rho \mu)=e^{i \omega_{\mathrm{VIS}} t}\left[\left(\mu_{01} \rho_{10}\right.\right.$ $\left.\left.-\mu_{10} \rho_{01}\right)+\left(\mu_{12} \rho_{21}-\mu_{21} \rho_{12}\right)\right]$. The first two terms contain the signal at the fundamental transition $\omega_{01}$ and the second terms give rise to the signal at $\omega_{12}$, the so-called hot-band transition $(v=1 \rightarrow 2)$. Here, we assume that the VISupconversion pulse is continuous wave. We account for the spectral width of the upconversion pulse and the instrumental resolution by convoluting the resultant spectra with a 5 $\mathrm{cm}^{-1}$ broad Gaussian. Initially, the vibrational lifetime $T_{1}$ of both excited states was set to be $2.0 \mathrm{ps}$ in the calculations, as determined from previous time-resolved measurements for $\mathrm{CO}$ on other metal surfaces, ${ }^{12,13}$ and from extrapolation of the linewidth to $0 \mathrm{~K}^{15}$

The SFG light associated with the $v=1 \rightarrow 2$ hot band transition is due to the interaction of the visible field with the polarization $\rho_{12}$. It is clear from the above set of equations that the pathway for generating $\rho_{12}$ is $\rho_{00} \rightarrow \rho_{01} \rightarrow \rho_{11} \rightarrow \rho_{12}$, each step comprising of one interaction with the incident IR field [note that in Eqs. (2)-(6) each of these terms has the preceding term multiplied by the infrared field $(V)$ as a source term]. Hence, the polarization represented by $\rho_{12}$ is of third order, and the additional interaction with the visible field makes it a fourth-order nonlinear optical process. A second pathway leading to $\rho_{12}$ proceeds via the overtone polarization $\rho_{02}: \rho_{00} \rightarrow \rho_{01} \rightarrow \rho_{02} \rightarrow \rho_{12}$. By setting either one of the source terms in Eq. (5), i.e., $i V_{12}\left(\tilde{\rho}_{11}-\tilde{\rho}_{22}\right)$ or $i V_{10} \tilde{\rho}_{02}$, to zero, it is possible to distinguish between the relative contributions of the two pathways. It turns out that the contribution via the overtone polarization $\rho_{02}$ amounts to only $1 \%$ of the overall $\rho_{12}$; the pathway via population in $v=1\left(\rho_{11}\right)$ is dominant.

The calculated resonant contribution to the spectra in Fig. 4 are shown as solid lines in the lower panel, and the agreement with results from the fit to the resonant SFG (dotted lines) are excellent. The time-dependent envelopes of the polarization at the fundamental $\left(P_{01}\right)$ and the hot-band $\left(P_{12}\right)$ transition are depicted in the upper panel of Fig. 5 for the 

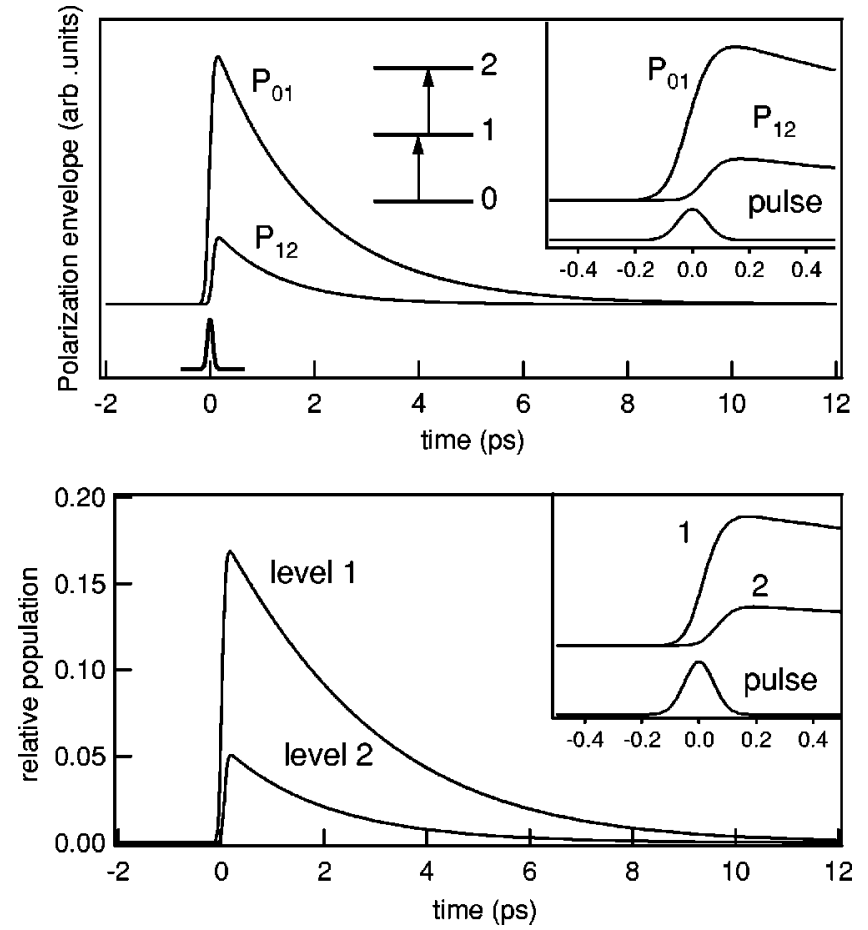

FIG. 5. Upper panel: Time-evolution of the polarization at the fundamental $\left(P_{01}\right)$ and hot-band $\left(P_{12}\right)$ transition. These calculations correspond to the 11 $\mu \mathrm{J}$ data of Fig. 4. The insets depicts the same data around time zero. Note the delayed rise of $P_{12}$ compared to $P_{01}$. The pulse envelope is depicted around zero delay. Lower panel: Time-evolution of excited state population in the first and second excited state levels.

calculation that reproduces the $11 \mu \mathrm{J}$-experiment. The lower panel depicts the time evolution of the population in the first and second excited states. Note that, as expected, the rise of the polarization at the hot-band transition $P_{12}$ is delayed with respect to that of the fundamental $P_{01}$. This is due to the fact that the buildup of the polarization $P_{12}$ arises from a sequence of interactions with the electromagnetic field: the initial interaction creates polarization $P_{01}$, and subsequently an additional interaction causes population to be transferred to $v=1$. The third interaction with the IR field gives rise to $P_{12}$. The final, nonresonant interaction with the visible field creates the SFG from this vibrational polarization. For the same reason the population in $v=2$ rises delayed when compared to the population in the first excited state (lower panel of Fig. 5). Approximately $15 \%$ of the CO molecules excited to the first vibrationally excited state, and about $5 \%$ to the second vibrationally excited state. The relatively large amount of population in $v=2$ is due to the larger transition dipole moment associated with the $1 \rightarrow 2$ transition compared to the fundamental.

The integrated intensity of the hot-band compared to that of the fundamental $\left(I_{12} / I_{01}\right)$ depends on the precise value of the vibrational lifetime $T_{1}$; the degree of saturation increases with increasing lifetime for a given excitation density. This is demonstrated in Fig. 6 which depicts $\left(I_{12} / I_{01}\right)$ as a function of IR fluence. However, since the duration of our laser pulses $(120 \mathrm{fs})$ is appreciably shorter than the typical relaxation times ( $T_{1}$ and $T_{2} \sim 1 \mathrm{ps}$ ), the effect does not allow for an accurate estimates of the lifetime given the error bars asso-

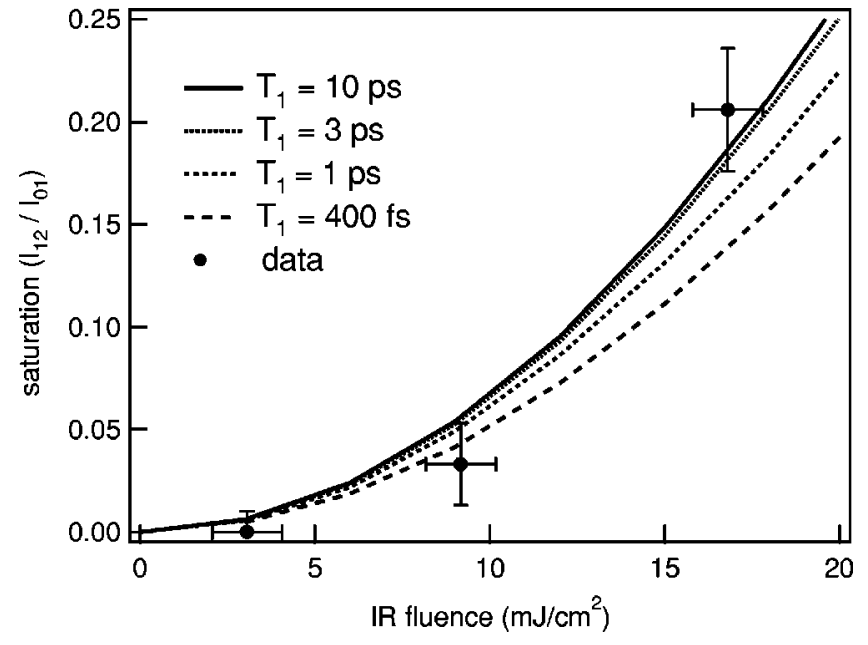

FIG. 6. Relative intensity of the hot-band compared to the fundamental transition as a function of incident IR fluence. The experimentally determined fluence has a systematic uncertainty, and was scaled by a factor 1.1 for the data to coincide with calculations.

ciated with experimental data. We can therefore only conclude that the lifetime exceeds $\sim 0.5 \mathrm{ps}$ (where it should be noted that a sharper lower limit may be obtained from the IR linewidth at low temperatures ${ }^{15}$ ).

The efficiency of the population transfer to higher lying vibrational states may be substantially enhanced by chirping the infrared excitation pulses, as has been demonstrated, for example, for gas phase $\mathrm{NO},{ }^{8}$ and $\mathrm{W}(\mathrm{CO})_{6}$ in solution. ${ }^{9}$ The mechanism behind the enhancement of population transfer is that the frequency of the laser field is swept within the duration of the laser pulse, so that the appropriate (viz., resonant) frequency is offered to the system at all times during the pulse: the front of the pulse contains high-frequency components for the transitions near the ground state at the bottom of the potential well and the trailing edge of the pulse contains lower frequencies for transitions between higherline states (the potential is anharmonic). It is not clear in advance that this scheme works equally well for molecules on surfaces as it does in the $\operatorname{gas}^{8}$ and liquid 9 phase, since there are additional considerations on surfaces; in the gas phase, most relaxation processes are very slow, whereas on surfaces vibrational relaxation occurs on picosecond time scale. The application of a chirp necessarily lengthens the duration of the laser pulse for a given spectral bandwidth; hence there will be a competition between an increase in population transfer due to the presence of the chirp, and a decrease in efficiency since population can decay during the lengthened pulse. Here, we will briefly investigate the possibility of extending this technique to vibrations on surfaces.

One can use a pulse shaper to positively or negatively chirp pulses in the mid-infrared. The amount of chirp in the laser pulse can be characterized by $\alpha$, which is proportional to the second derivative of the phase of the light wave of center frequency $\omega_{0}$ (see, e.g., Ref. 37),

$$
\left.\alpha \propto \frac{\partial^{2} \varphi}{\partial^{2} \omega}\right|_{\omega=\omega_{0}}\left(\omega-\omega_{0}\right)^{2} .
$$




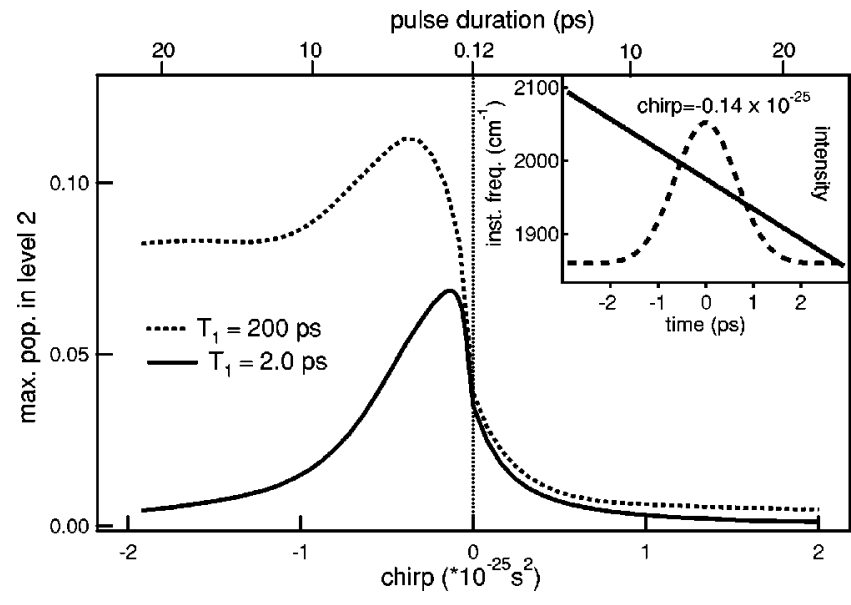

FIG. 7. Calculated population in the second excited state $v=2$ as a function of chirp. With negative chirp, the blue components within the bandwidth are offered to the $\mathrm{CO}$ molecules first, and more efficient population transfer takes place. The comparison between calculations for a realistic lifetime $\left(T_{1}=2 \mathrm{ps}\right.$, solid line $)$ and a very long lifetime $\left(T_{1}=200 \mathrm{ps}\right.$, dashed line $)$ demonstrate the competition between the vibrational ladder climbing and energy relaxation. The inset shows the pulse characteristics for the chirp where population transfer is maximal for $T_{1}=2 \mathrm{ps}$ : instantaneous frequency (left axis) and intensity envelope. Note that in the chirped pulse initially the blue part of the spectrum is present (resonant with the fundamental at $\sim 1990 \mathrm{~cm}^{-1}$ ), followed by the red part (resonant with the hot band at $\sim 1960 \mathrm{~cm}^{-1}$ ).

For a Gaussian laser pulse, the duration of the chirped pulse $\tau_{p}$, for an originally bandwidth-limited pulse of duration $\tau_{0}$ and bandwidth $\Delta \omega$ (full width at half maximum) is

$$
\tau_{p}^{2}=\tau_{0}^{2}+\left(\frac{8 \alpha \ln 2}{\tau_{0}}\right)^{2}
$$

and the instantaneous frequency $\omega(t)$ varies linearly with time,

$$
\omega(t)=\omega_{0}+\frac{\alpha\left(\frac{\Delta \omega^{2}}{\ln 2}\right)^{2}}{8+2 \alpha^{2}\left(\frac{\Delta \omega^{2}}{\ln 2}\right)^{2}} t .
$$

We have calculated the maximal population in the second excited state $v=2$ as a function of chirp solving Eqs. (1)-(5) without the rotating frame approximation, since the frequency now varies during the laser pulse.

The result of this calculation for an IR fluence of $10 \mathrm{~mJ} /$ $\mathrm{cm}^{2}$ are depicted in Fig. 7, and it is clear that the population transfer is indeed more efficient for a slightly negatively chirped pulse. As expected, providing the resonance frequency for the $(v=1 \rightarrow 2)$ following the $(v=0 \rightarrow 1)$ frequency can increase the efficiency of population transfer to $v=2$. A factor of 2 with respect to the transform limited pulses can be gained with a chirp of $\alpha=-1.36 \times 10^{-26} \mathrm{~s}^{2}$, resulting in a pulse duration of 1.5 ps (see inset of Fig. 7; original pulse duration: $120 \mathrm{fs}$ ). Conversely, a positive chirp leads to a strong decrease in population transfer. The fact that optimal population transfer is achieved for a pulse duration similar to the energy relaxation lifetime, indicates that there is a large effect of the rapid vibrational relaxation at the metal surface: Indeed, as shown by the dotted line in Fig. 7,

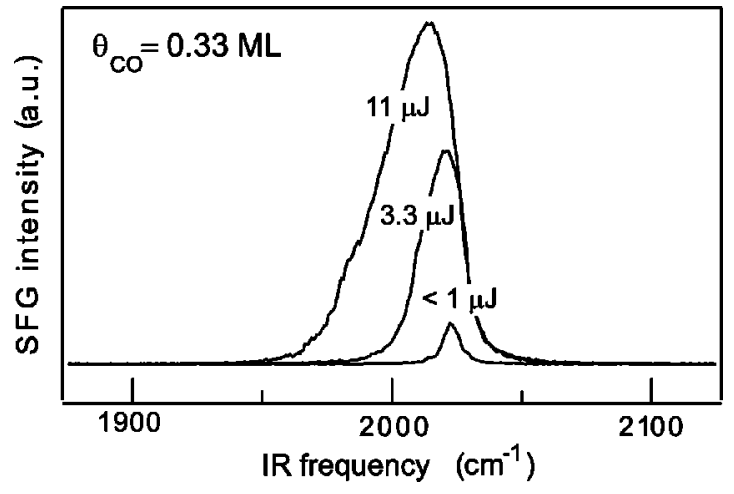

FIG. 8. SFG spectra of the CO-stretch vibration of $(\sqrt{3} \times \sqrt{3})-\mathrm{CO} / \mathrm{Ru}(001)$ $(\theta=0.33 \mathrm{ML})$ as a function of IR energy at $95 \mathrm{~K}$. Note the absence of discrete vibrational transitions that are present for low CO coverage (see Figs. 1 and 4).

if the vibrational lifetime is increased by two orders of magnitude to $200 \mathrm{ps}$, a 4-fold increase in population transfer can be obtained for $\alpha=-4.010^{-26} \mathrm{~s}^{2}$ (corresponding pulse duration: $4.5 \mathrm{ps}$ ). For practical applications, these results indicate that, for vibrations on surfaces, the losses associated with shaping an infrared pulse $(\sim 30 \%)$ may cancel out some of the gain that can be achieved by doing so, although the relative gain may be significantly higher for higher-lying vibrational states. In addition, there may be other modes or other adsorbates that have longer lifetimes, even on metal surfaces.

\section{VIBRATIONAL ENERGY TRANSFER}

In the previous paragraph excited state SFG spectra were presented at relatively low $\mathrm{CO}$ coverages. This was done to avoid the effects of intermolecular interactions between the $\mathrm{CO}$ molecules, which is known to be present at higher coverages. Extensive and detailed linear infrared spectroscopic investigations (reflectance absorption infrared spectroscopy) of the $\mathrm{C}-\mathrm{O}$ stretch vibration of $\mathrm{CO}$ on $\mathrm{Ru}(001)$ have demonstrated that with increasing coverage pronounced shifts (frequency renormalization) and narrowing of the vibrational resonance occurs. ${ }^{15}$ This is due to the fact that there exists strong dipolar coupling between the $\mathrm{CO}$ molecules, effectively delocalizing the vibrational excitation. This means that the discrete level picture of Fig. 1 breaks down and should be replaced by a band structure. ${ }^{38}$

Indeed, at higher coverages very different excited state SFG spectra are obtained. For $0.33 \mathrm{ML}$, no hot band is observed - there are no longer discrete vibrational levelsbut as shown in Fig. 8 increasing IR power leads to a broadening and red-shift of the resonance as a result of saturation. The excited-state spectra show a single broadened feature with a non-Lorentzian line shape and an asymmetric tail to the long-wavelength side. These observations can only be explained by taking into account $\mathrm{CO}-\mathrm{CO}$-interactions in addition to the anharmonicity of individual $\mathrm{CO}$ molecules. At 0.33 ML dipole-dipole coupling between the adsorbed molecules leads to a delocalization of vibrational energy within the $\mathrm{CO}$ adlayer. According to Shen et al. ${ }^{39}$ the observed redshift can be related to the number of excited $\mathrm{CO}$ molecules, 


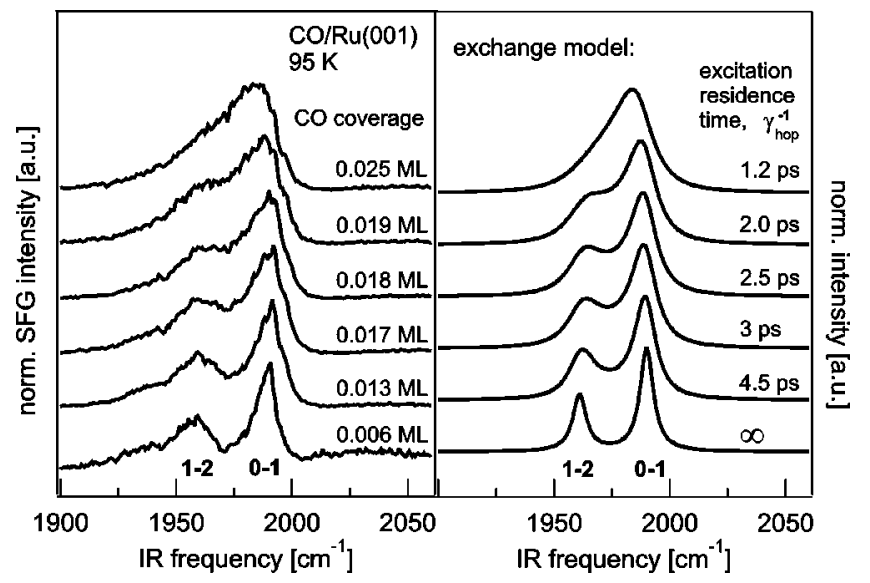

FIG. 9. Left panel: SFG spectra of the $\mathrm{CO}$ stretch as a function of $\mathrm{CO}$ coverage at $95 \mathrm{~K}$. With increasing coverage the $v=1 \rightarrow 2$ hot band decreases and disappears at a coverage of $0.025 \mathrm{ML}$. Right panel: Prediction of the exchange model for varying excitation residence times.

or rather, the average degree of excitation per $\mathrm{CO}$ molecule, since the excitation is shared coherently by the $\mathrm{CO}$ molecules. In the limit of strong intermolecular interaction, the red-shift equals $4 C \Gamma$, with $C$ the number of excited phonons per $\mathrm{CO}$ molecule and $\Gamma$ the vibrational anharmonicity [13.6 $\mathrm{cm}^{-1}$ (Refs. 29, 36)]. At the highest degree of excitation, a shift of $-9 \mathrm{~cm}^{-1}$ is observed, corresponding to $C=0.17$, in good agreement with the excited state population observed at low coverage (see above).

The difference between the excited state spectra at low and high coverage are due to the fact that at low coverage a fraction $(\sim 15 \%)$ of $\mathrm{CO}$ molecules is in $v=1$, whereas the rest of the molecules remain in the ground state. At high coverage, the excitation is shared by all the molecules and by adding energy to the system, the vibrational amplitude of all molecules is increased. Thus with increasing coverage a transition from a localized vibration to a delocalized phonontype excitation must occur. Figure 9 depicts the measurements of this transition (left panel). On the right panel of this figure are calculations that reproduce the experimentally observed spectra. The experimental SFG spectra were recorded at a temperature of $95 \mathrm{~K}$ for increasing $\mathrm{CO}$ coverage (viz., for increasingly strong intermolecular coupling). With increasing coverage the $v=1 \longrightarrow 2$ hot band gradually disappears, indicating a transition from discrete vibrational energy levels to a continuum of states: the delocalization of vibrational energy. This transition seems to be complete at a $\mathrm{CO}$ coverage as low as $0.025 \mathrm{ML}$, where the hot-band is no longer discernible. The continuous character of the transition is in agreement with theoretical predictions ${ }^{39}$ and previous observations in three-dimensional systems. ${ }^{40,41}$

The calculated spectra in the right panel of Fig. 9 result from a model which describes the energy delocalization as a function of finite residence time of the $(v=1 \rightarrow 2)$ excitation on one oscillator, before hopping to a neighboring oscillator. This means that the molecule will exhibit the spectrum associated with the $0 \rightarrow 1$ transition as long as it is in the ground state. Once the excitation finds this particular molecule ("hops" onto it), its spectrum will be that of the $1 \rightarrow 2$ transition. In this manner, the $0 \rightarrow 1$ frequency and the $1 \rightarrow 2$ frequency become mixed, and the observed overall spectrum will then critically depend on the speed at which the mixing occurs, viz., the hopping rate. This process of spectral modulation is very similar to that determining the vibrational line shapes in the condensed phase through so-called exchange. ${ }^{42}$ The exchange model by Shelby et al. $^{42}$ describes the effects of thermal excitation and de-excitation of a low-frequency mode $(\Omega)$, which is anharmonically coupled to a highfrequency vibration $(\omega)$. The anharmonic coupling leads to a frequency difference $\delta \omega / 2$ between the $\left\langle n_{\Omega}=0 ; n_{\omega}=0\right|$ $\rightarrow\left|n_{\Omega}=0 ; n_{\omega}=1\right\rangle$ and the $\left\langle n_{\Omega}=1 ; n_{\omega}=0|\rightarrow| n_{\Omega}=1 ; n_{\omega}\right.$ $=1\rangle$ transition (we will refer to these as the $\langle 0,0|$ $\rightarrow|0,1\rangle$ and the $\langle 1,0|\rightarrow| 1,1\rangle$ transitions); $\delta \omega$ is a measure of the anharmonic coupling strength between the two modes. The spectrum associated with the high-frequency vibration is determined by the rate of energy exchange between the lowfrequency mode and the bath, i.e., the rate of excitation and de-excitation rate of mode $\Omega$. These rates are given by $W_{+}$ $=\gamma \bar{n}(\Omega)$ and $W_{-}=\gamma(\bar{n}(\Omega)+1)$, respectively, with $\bar{n}(\Omega)$ $=\left[e^{\hbar \Omega / k_{b} T}-1\right]^{-1}$ the average number of reservoir excitations at frequency $\Omega$, and $\gamma$ the lifetime of the lowfrequency mode. The expression for the resulting absorption line was derived to be $\mathrm{b}^{42}$

$$
I(\omega)=\frac{W_{+}(\delta \omega)^{2} /\left(1+W_{+} / W_{-}\right)}{\left[\omega^{\prime 2}-(\delta \omega / 2)^{2}\right]^{2}+\left[W_{-}\left(\omega^{\prime}+\delta \omega / 2\right)+W_{+}\left(\omega^{\prime}-\delta \omega / 2\right)\right]^{2}},
$$

where $\omega^{\prime}=\omega-\omega_{0} \delta \omega / 2$ (with $\omega_{0}$ the fundamental transition).

Analogous, in our system, we can identify $\langle 0,0|\rightarrow| 0,1\rangle$ as the $v=0 \rightarrow 1$ and $\langle 1,0|\rightarrow| 1,1\rangle$ as the $v=1 \rightarrow 2$ transition; the rate at which an oscillator changes frequency is determined by the residence time of the excitation on one molecule. We can therefore use the above expression for the spectrum by expressing the parameters $W_{+}, W_{-}, \gamma$ and $\delta \omega$ occurring in Eq. (14) in terms of the hopping rate $\gamma_{\text {hop }}$ (the inverse of the excitation residence time), the ratio of excited to nonexcited oscillators $n_{\text {exc }}$ and the anharmonicity of the $\mathrm{C}-\mathrm{O}$ stretch vibration $\Gamma$, as

$$
\begin{aligned}
& W_{+}=\gamma_{\text {hop }} n_{\mathrm{exc}}, \\
& W_{-}=\gamma_{\mathrm{hop}}, \\
& \delta \omega=2 \Gamma,
\end{aligned}
$$




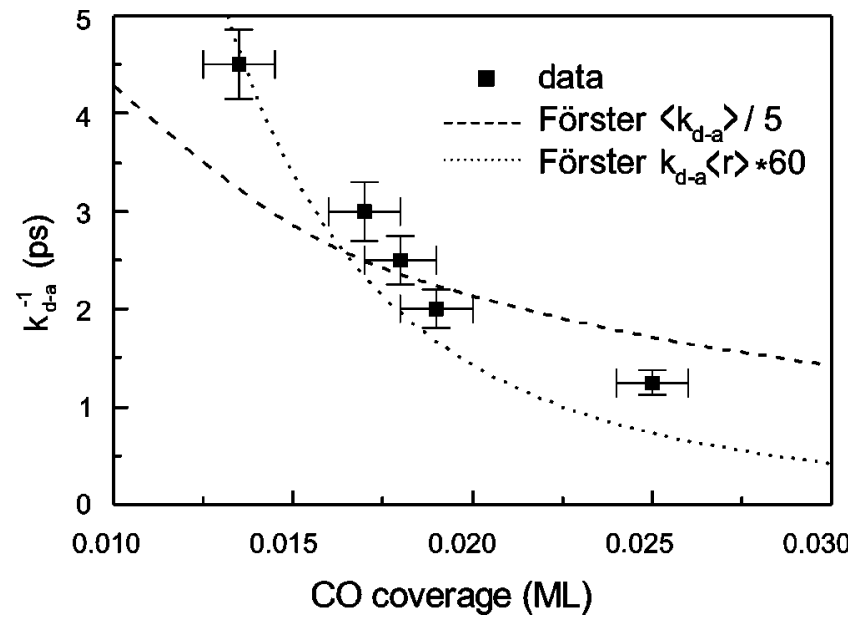

FIG. 10. Experimental (solid boxes) and calculated (lines) coveragedependent energy transfer times $\left(k_{d \rightarrow a}\right)^{-1}$ as a function of coverage $\theta$ of $\mathrm{CO}$ molecules on the $\mathrm{Ru}(001)$ surface. The dotted line represents the onedimensional calculation with $r=\langle r\rangle$, the dashed line employing the discretized 2D radial distribution function $g(r)$ (see text). Scaling factors are indicated in the legend.

so that $n_{\text {exc }} /\left(n_{\text {exc }}+1\right)$ is the fraction of excited $\mathrm{CO}$ molecules. The fact that the transition dipole moment for the hot-band $\mu_{12}$ is approximately twice that of the fundamental, can be accounted for in this model by defining an effective "downrate" $W_{-}^{\text {eff }}=0.5 \times \gamma_{\text {hop }}$, and setting $W_{-}=W_{-}^{\text {eff }}$ in Eq. (14). This increases the intensity of the $v=1 \rightarrow 2$ transition at frequency $\left(\omega^{\prime}-\delta \omega / 2\right)$ by a factor of 2 , and is correct for $n_{\text {exc }} \geq 0.05$, and considering we are interested only in normalized spectra. Lifetime broadening along with the instrumental response function are included by convoluting the resulting spectra with a $7 \mathrm{~cm}^{-1}$ Lorentzian. Since $\Gamma$ and $n_{\text {exc }}$ can be obtained directly from low-coverage data of the left panel, the only adjustable parameter in the model is the excitation residence time $\gamma_{\text {hop }}^{-1}$. For this dataset, $\Gamma=14.5$ and $n_{\text {exc }}=0.25$ (corresponding to a fraction of excited oscillators of $20 \%$ ). With increasing coverage the intermolecular coupling increases and therefore the excitation residence time decreases. As can be seen from Fig. 9, the model describes the data remarkably well: Both the gradual filling of the gap between the two transitions as well as the red-shift of the resonance frequency of the fundamental transition as a result of increasing energy exchange are reproduced by varying $\gamma_{\text {hop }}$ between infinity and $1.2 \mathrm{ps}$. The increased hopping rate at higher coverages gives rise to motional narrowing of the two absorption lines, that are observed to merge together with increasing coverage. Figure 10, depicting the fitted energy transfer rates versus surface coverage, summarizes the fits. ${ }^{43}$

The dipole-dipole interactions between $\mathrm{CO}$ molecules on the surface of $\mathrm{Ru}(001)$ gives rise to energy transfer of vibrational excitations. This is the so-called first Förster energy transfer, ${ }^{44}$ which is well-known for electronic systems ${ }^{45-47}$ and has recently been observed at a surface among different $\mathrm{Si}-\mathrm{H}$ stretch vibrations for hydrogen adsorbed on a stepped silicon surface. ${ }^{17-19}$ The expression for the rate of energy transfer $k_{d \rightarrow a}$ from an excited donor dipole to an unexcited acceptor dipole for a Förster-type interaction reads $^{48}$

$$
k_{d \rightarrow a}=\frac{\kappa^{2} \mu_{d}^{2} \mu_{a}^{2}}{4 n^{4} \varepsilon_{0}^{2} h^{2} c r^{6}} \int_{0}^{\infty} g_{d}(\nu) g_{a}(\nu) d \nu .
$$

In this expression, $\varepsilon_{0}, h$, and $c$ are the vacuum permittivity, Planck's constant and the velocity of light, respectively. $\mu_{d}$ and $\mu_{a}$ are the absolute values of the transition dipole moments of donor and acceptor, $2.1 \times 10^{-30} \mathrm{C} \mathrm{m}$ for $\mathrm{CO}$ on $\mathrm{Ru}$ at low coverages (including the image dipole). The distance between the dipoles is given as $r, n$ is the refractive index of the medium between the dipoles and $g_{d}(\nu)$ and $g_{a}(\nu)$ are the normalized homogeneous line shapes of the donor and acceptor vibrations, respectively. The effect of the relative orientations of the dipoles is taken into account by the orientation factor $\kappa^{2}\left(0 \leqslant \kappa^{2} \leqslant 4\right)$, given by $\kappa=\mathbf{e}_{d} \cdot \mathbf{e}_{a}-3\left(\mathbf{e}_{\mathrm{d}} \cdot \mathbf{r}\right)$ $\times\left(\mathbf{e}_{\mathbf{a}} \cdot \mathbf{r}\right) /|\mathbf{r}|^{2}$, with $\mathbf{e}$ the unit vectors parallel to the donor and acceptor dipoles and $\mathbf{r}$ the vector joining the dipoles $(|\mathbf{r}|=r)$. For parallel dipoles perpendicular to the surface considered here, $\kappa=1$.

For the homogeneous system of $\mathrm{CO} / \mathrm{Ru}(001)$ the overlap integral between the two normalized Lorentzians which have an equal full width at half maximum (FWHM) $\delta \nu_{\text {hom }}$ and are centered at the same frequency, equals

$$
\int_{0}^{\infty} g_{d}(\nu) g_{a}(\nu) d \nu=\frac{1}{\pi \delta \nu_{\mathrm{hom}}} .
$$

For low coverages at low temperatures $(\sim 100 \mathrm{~K}), \delta \nu_{\text {hom }}$ $=5 \mathrm{~cm}^{-1} \cdot{ }^{15}$ Inserting these values into Eq. (1) and setting $n=1.0$ (refractive index of vacuum), we obtain

$$
k_{d \rightarrow a}=\frac{3.01 \times 10^{17}}{r^{6}},
$$

with $r$ in $\AA$. For the well-ordered $\sqrt{3} \times \sqrt{3}$-structure the distance between two neighboring CO molecules is $r=4.7 \AA$, resulting in $k_{d \rightarrow a}=(36 \mathrm{fs})^{-1}$. This means that at high coverages the coherent energy transfer occurs on a time scale comparable to the vibrational motion of the $\mathrm{CO}$ molecules (the frequency of $2000 \mathrm{~cm}^{-1}$ corresponds to a vibrational period of $17 \mathrm{fs}$ ). In this limit, it is more appropriate to view the excitation as coherently delocalized over many coupled $\mathrm{CO}$ molecules, rather than as hopping from one $\mathrm{CO}$ molecule to the next ${ }^{15}$ (note that, since the energy transfer is coherent, this does not lead to dephasing of the vibrational transition and the associated line-broadening). At lower coverages, the hopping picture is appropriate and we can calculate an average distance $\langle r\rangle$, from which a value of the hopping rate $k_{d \rightarrow a}^{\langle r\rangle}$ can be calculated. For example, for $\theta=0.025 \mathrm{ML}$, $\langle r\rangle=17 \AA$ and $k_{d \rightarrow a}^{\langle r\rangle}=(80 \mathrm{ps})^{-1}$, which is much slower than the $(1.2 \mathrm{ps})^{-1}$ from the fit. Although the absolute numbers for the hopping rate are off significantly, the coverage dependence of the hopping rate is reasonably reproduced by this simple model, as evidenced by the dotted line in Fig. 10.

However, at low coverages there will be a statistical distribution of intermolecular distances on the surface, and due to the $r^{6}$-dependence of the transfer rate, there will be a relatively large contribution from molecules close to each other. We can account for this by noting that for an arbitrary 


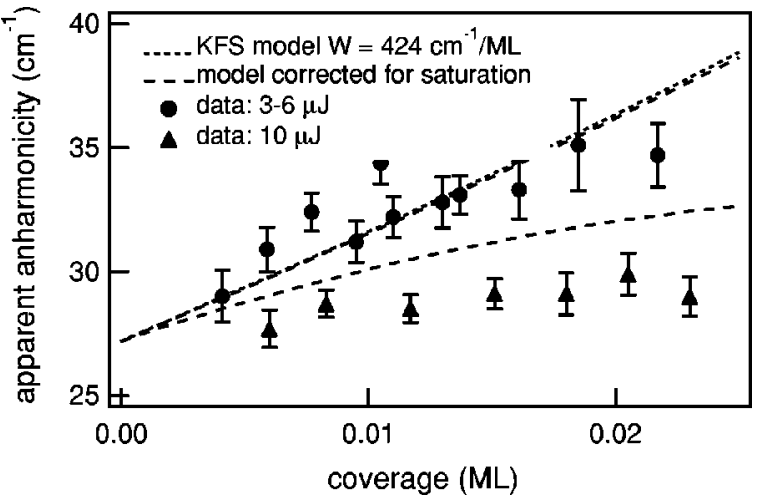

FIG. 11. The frequency difference between the $v=0 \rightarrow 1$ and the $v=1$ $\rightarrow 2$ transitions (apparent anharmonicity, or two-phonon bound state frequency) as a function of $\mathrm{CO}$ surface coverage for low (circles) and high (triangles) incident IR energies. The dotted line is the result of the calculation according to the KFS-theory described in the text. Dashed lines are the same calculation corrected for saturation effects, observed to be large for high fluences, but negligible for the lowest fluences used.

pair-distribution function $g(r)$, the number of acceptors around an excited donor in the area delimited by the circles with radius $r$ and $r+d r$ is given by $2 \pi r \partial r \rho g(r)$, with $\rho$ the density (per surface area) of CO molecules. The density $\rho$ is a function of the coverage $\theta$, given by $\rho=0.158 \times \theta$ (in $\AA^{-2}$ ). Thus, the effective rate of transfer, averaged over the distribution of molecules reads

$$
\left\langle k_{d \rightarrow a}\right\rangle=\int_{0}^{\infty} 2 \pi r d r \rho g(r) k_{d \rightarrow a} .
$$

We can consider the discretized $g(r)$, since we know for the hexagonal structure of the $\mathrm{Ru}(001)$ surface, that there are $N_{i}$ sites for the $i$ th "lattice site" at a distance $r_{i}$. For the first 11 shells, $N_{i}=6,6,6,12,6,6,12,6,12,12$, and 6 , for $r_{i}$ $=r_{0}, \sqrt{3} r_{0}, 2 r_{0}, \sqrt{7} r_{0}, 3 r_{0}, 2 \sqrt{3} r_{0}, \sqrt{13} r_{0}, 4 r_{0}, \sqrt{19} r_{0}$, $\sqrt{21} r_{0}$, and $5 r_{0}$, respectively, with $r_{0}$ the distance between two nearest-neighboring lattice sites $(2.71 \AA)$. The average transfer rate calculated in this way will be denoted by $\left\langle k_{d \rightarrow a}\right\rangle$ and is given by

$$
\left\langle k_{d \rightarrow a}\right\rangle=\sum_{i=2}^{11} \theta N_{i} k_{\mathrm{d} \rightarrow \mathrm{a}}\left(r_{i}\right),
$$

with $k_{d \rightarrow a}\left(r_{i}\right)$ defined in Eq. (20) and $\theta$ the CO coverage, which represents the possibility of finding a $\mathrm{CO}$ molecule on a $\mathrm{Ru}$ atom. The sum is taken starting with $i=2$, since two neighboring $\mathrm{Ru}$ atoms cannot both accommodate a $\mathrm{CO}$ molecule; at low coverage the minimal distance between two $\mathrm{CO}$ molecules is $\sqrt{3} \times 2.71=4.7 \AA$. Truncating the distribution at $i=11$ results in an error smaller than $1 \%$, due to the strong $r$-dependence of $k_{d \rightarrow a}\left(r_{i}\right)$. Note that expression (22) can be written as $\left\langle k_{d \rightarrow a}\right\rangle=k_{0} \theta$, with $k_{0}=1.3 \times 10^{14} \mathrm{~s}^{-1}$.

This procedure results in the value for $\left\langle k_{d \rightarrow a}\right\rangle$ $=(0.3 \mathrm{ps})^{-1}$, at a coverage $\theta=0.025 \mathrm{ML}$, appreciably smaller than that obtained from the average distance. This value is in very reasonable agreement with the $(1.2 \mathrm{ps})^{-1}$ obtained from the fit, especially considering that there are no adjustable parameters. The coverage dependence of $\left\langle k_{d \rightarrow a}\right\rangle$, exhibiting a proportionality $k \propto \theta$, also agrees reasonably well with experimental data. In particular, the coverage dependence is best described by $\left\langle k_{d \rightarrow a}\right\rangle=k_{0} \theta$, with $k_{0}=2.6$ $\times 10^{13} \mathrm{~s}^{-1}$ (a factor of 5.0 smaller than the calculated value).

This linear dependence of the exchange rate on the coverage is also in good agreement with observations of temperature dependent lineshift of the $\mathrm{C}-\mathrm{O}$ stretch vibration at various coverages with reflectance absorption infrared spectroscopy (RAIRS). In these experiments, the dynamic dipole shift, which is a qualitative measure for the degree of delocalization of the $\mathrm{C}-\mathrm{O}$ stretch vibration, was found to increase linearly with coverage. ${ }^{15}$

\section{SUMMARY AND CONCLUSIONS}

To summarize, femtosecond vibrational saturation spectroscopy of the $\mathrm{CO}$-stretch vibration of $\mathrm{CO} / \mathrm{Ru}(001)$ allows for the study of the dynamics of the CO-stretch vibration at $\mathrm{CO}$ coverages as low as $0.01 \mathrm{ML}$. The strong excitation (saturation) of the fundamental transition of the CO-stretch make the simultaneous observation of the fundamental and subsequent $v=1 \rightarrow 2$ hot band transition possible. At low coverages, the vibrational excitation is localized on individual $\mathrm{CO}$ molecules, and it is demonstrated that [an approximate] $\sim$ twofold increase in the $v=2$ excited state population can be achieved by chirping the broadband infrared laser pulse. At higher $\mathrm{CO}$ coverages, a transition from localized oscillators to delocalized phonons can be observed directly. The $v=1 \rightarrow 2$ hot-band transition of the $\mathrm{CO}$-stretch vibration serves as a sensitive indicator for vibrational energy localization. With increasing coverage the lateral interactions between the adsorbed molecules and therefore the delocalization of vibrational energy increases which leads to the disappearance of the hot band at a coverage around 0.025 ML. In conjunction with a modified exchange model, the dynamics of the delocalization process can be deduced, and the excitation is found to "hop around" on time scales down to $1.2 \mathrm{ps}$, further decreasing with increasing coverage. These excitation residences times can be reproduced by a simple model of resonant vibrational energy transfer.

\section{ACKNOWLEDGMENTS}

The authors are very grateful to P. Jakob for continuous help throughout this study, to P. Guyot-Sionnest and A.W. Kleyn for many helpful discussions, and to S. Roke for careful and critical reading of the manuscript. This work was supported in part by a fellowship for (M.B.) of the Royal Netherlands Academy of Arts and Sciences and by the Deutsche Forschungsgemeinschaft through SFB 450.

\section{APPENDIX: SATURATION EFFECTS}

Throughout the experimental studies described in this paper, we have employed the fact that we can saturate the $\mathrm{C}-\mathrm{O}$ stretch vibration, i.e., excite a significant fraction of these oscillators to their first excited state, to obtain information on the vibrational dynamics. In this Appendix, we will briefly describe the unwanted side-effects that may appear using these techniques, and must be accounted for carefully.

The dipole-dipole coupling among the $\mathrm{CO}$ molecules gives rise to the delocalization of vibrational energy, and the 
dispersion of the resulting delocalized phonon-mode caused by this intermolecular interaction can be observed. ${ }^{38}$ It also leads to an increase in apparent anharmonicity (the difference in frequency between the $v=0 \rightarrow 1$ and $v=1 \rightarrow 2$ transition) with increasing coverage. Figure 11 depicts the apparent anharmonicity versus the surface coverage. This quantity is sometimes also referred to as two-phonon bound state (TPBS) shift, ${ }^{12,39}$ and is a measure of the effective dispersion at low coverage. Due to the interaction between the molecules at the surface, the energy difference increases with increasing interaction strength (coverage) in accordance with theory. ${ }^{39}$ The $(0 \rightarrow 1)$ and the $(1 \rightarrow 2)$ transition frequencies are derived from a least-squares fits to a set of experimental data taken at low IR energies (3-6 $\mu \mathrm{J})$ in order to minimize the effect of saturation, and a set obtained at $10 \mu \mathrm{J}$. Note that there is a large difference in the apparent anharmonicities for these different energies, implying that saturation effects have to be taken into account when considering the data. The dotted line in this figure is a prediction from a model by Kimball, Fong and Shen (KFS model) ${ }^{39}$ assuming a system of coupled anharmonic oscillators defined by anharmonicity $\Gamma$ and effective dispersion $W$, which is a measure of the intermolecular interaction. $W$ is treated as a free parameter assuming a linear increase as a function of $\mathrm{CO}$ coverage. Although Ref. 39 does not provide an explicit expression for a 2D system, the expressions for a one-dimensional linear chain and a 3D lattice with a semi-circular density of states results in very similar dispersions. ${ }^{12}$ To obtain the best agreement between the experimental data at low excitation energies experiment and theory $W$ has to increase by 424 $\mathrm{cm}^{-1} / \mathrm{ML}$ and $405 \mathrm{~cm}^{-1} / \mathrm{ML}$ at these low coverages, for the 3D and 1D model, respectively. These values are very satisfactory, since it means that at $0.025 \mathrm{ML}$ coverage where roughly the phonon-localization transition is observed (which is theoretically predicted to take place when $\Gamma$ $\approx W)$, the effective dispersion amounts to approximately $(0.025 \times 410) \mathrm{cm}^{-1}=10.3 \mathrm{~cm}^{-1}$, which is very close to the anharmonicity of $13.6 \mathrm{~cm}^{-1}$.

It is clear that the effective dispersion (or, equivalently, the apparent anharmonicity) is a quantity inherent to the system, and should not depend on the energy of the pulses used to establish it. Apparently, at an infrared energy of $10 \mu \mathrm{J}$ the distortion due to saturation is very large (a least square fit to those data results in a dispersion 3.5 times smaller than that obtained from the low-energy data). The question that arises is to what extent the $\sim 3.5 \mu \mathrm{J}$-measurements also suffer from saturation effects.

To investigate the effect of saturation on this measurement, and in particular whether the low-energy data set was recorded at sufficiently low energy to obtain an accurate value for the dispersion, we use the exchange model that was introduced above. The exchange model, which can be used to calculate excited state spectra, does not incorporate the increase in apparent anharmonicity. Rather, it predicts that with (i) increasing coverage and (ii) increasing degree of excitation, the $(0 \rightarrow 1)$ and the $(1 \rightarrow 2)$ transitions will approach each other, finally merging together into one resonance, as can be observed in Fig. 9. Effect (i) is the physically interesting effect inherent to the vibrational dynamics

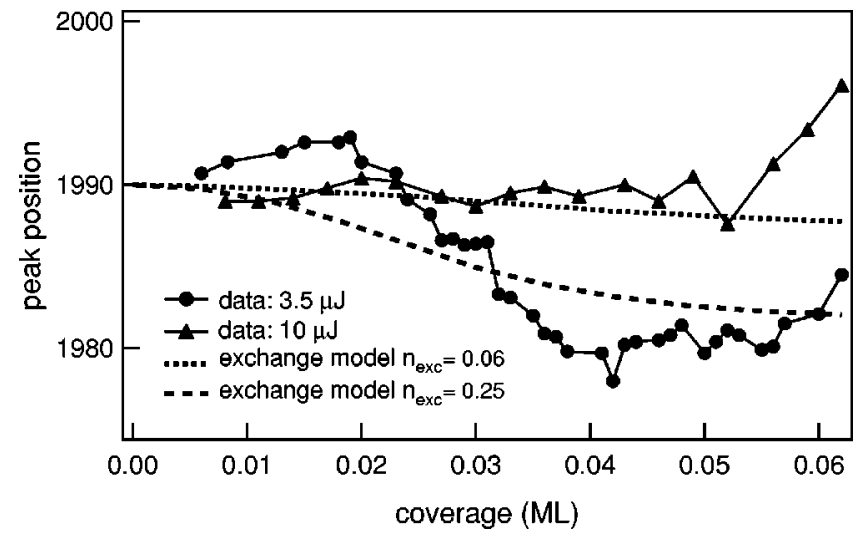

FIG. 12. Frequency of the fundamental $\mathrm{C}-\mathrm{O}$ stretch vibration as a function of coverage for two different excitation energies (triangles: $3.5 \mu \mathbf{J}$; circles: $10 \mu \mathbf{J})$. The lines are the results of calculations that incorporate the effect of saturation (see text), with degrees of excitation $n_{\text {exc }}$ denoted in the legend.

of the system: with increasing coverage the hopping rate will increase, resulting in an increased mixing of the $(0 \rightarrow 1)$ and the $(1 \rightarrow 2)$ state, leading to a decrease in apparent anharmonicity. Effect (ii) is an artifact of the technique: with increasing excitation, there will simply be more intensity of the $(1 \rightarrow 2)$ state to mix in with the fundamental transition, which will also result in the merging of the two lines: this means that as the excitation density increases, the two lines approach each other and the apparent anharmonicity decreases, although the coverage and therefore the hopping rate remains unchanged; this is an artifact of the measurement due to saturation.

This effect is illustrated in Fig. 12, which depicts the peak position of the fundamental $(0 \rightarrow 1)$ transition as a function of coverage for the two energies; at low energy the frequency is constant, whereas at higher energies, the fundamental transition is shifted to the red as dipole-dipole coupling sets in with increasing coverage. In other words, at high excitation densities the $(0 \rightarrow 1)$ transition is no longer purely $(0 \rightarrow 1)$, but has significant $(1 \rightarrow 2)$ mixed in, causing the red-shift. This effect is an artifact of the measurement and can be reproduced theoretically: the lines in this graph result from calculations with hopping rates $\left\langle k_{d \rightarrow a}\right\rangle=k_{0} \theta$, with $k_{0}=1.3 \times 10^{13} \mathrm{~s}^{-1}$, in agreement with the calculation presented in Fig. 10. The values of $n_{\mathrm{exc}}=0.06$ and $n_{\mathrm{exc}}$ $=0.25$ (corresponding to excited state fractions of 0.06 and 0.2 , respectively) were obtained from the transient spectra. It is interesting to note that experimentally the red-shift occurs more suddenly and at a higher coverage than predicted theoretically. It is clear from figure 12 the effect of saturation can be quite significant at higher excitation energies, but that these effects can be reproduced and accounted for using the theoretical approach described above.

Returning to Fig. 11, we can use this same calculation to estimate the effect of saturation on the apparent anharmonicity measurement. In addition to the calculation where saturation is neglected (dotted lines), two dashed lines are shown depicting the result of the calculation corrected for the saturation at the two energies. It is immediately clear that for low energies the effect of saturation is negligible, but indeed, at higher energies, the calculation demonstrates that the in- 
crease in apparent anharmonicity with increasing coverage is partly canceled out by saturation effects. Note that saturation effects will not affect the hopping times as obtained from the exchange model, since the saturation effect is incorporated directly in the model through $n_{\text {exc }}$.

Summarizing, it is clear that in the interpretation of nonlinear infrared spectra of coupled vibrations at surfaces, saturation effects must be incorporated for a correct description of the vibrational dynamics. Saturation artifacts can be avoided by retaining low excitation densities.

${ }^{1}$ A. Sinha, M. C. Hsiao, and F. F. Crim, J. Phys. Chem. 92, 6333 (1990); 94, 4928 (1991).

${ }^{2}$ M. J. Bronikowski, W. R. Simpson, and R. N. Zare, J. Chem. Phys. 97, 2194 (1993); 97, 2204 (1993).

${ }^{3}$ R. B. Metz, J. D. Thoemke, J. M. Pfeiffer, and F. F. Crim, J. Chem. Phys. 99, 1744 (1993).

${ }^{4}$ S. Chelkowski, A. D. Bandrauk, and P. B. Corkum, Phys. Rev. Lett. 65, 2335 (1990).

${ }^{5}$ S. Chelkowski and A. D. Bandrauk, Chem. Phys. Lett. 186, 264 (1991).

${ }^{6}$ D. J. Maas, D. I. Duncan, A. F. G. van der Meer, M. J. van der Zande, and L. D. Noordam, Chem. Phys. Lett. 270, 45 (1997).

${ }^{7}$ S. M. Arrivo, T. P. Dougherty, W. T. Grubbs, and E. J. Heilweil, Chem. Phys. Lett. 235, 247 (1995).

${ }^{8}$ D. J. Maas, D. I. Duncan, R. B. Vrijen, W. J. van der Zande, and L. D. Noordam, Chem. Phys. Lett. 290, 75 (1998).

${ }^{9}$ V. D. Kleiman, S. M. Arrivo, J. S. Melinger, and E. J. Heilweil, Chem. Phys. 233, 207 (1998).

${ }^{10}$ H. C. Chang and G. E. Ewing, Phys. Rev. Lett. 65, 2125 (1990).

${ }^{11}$ M. Bonn, R. A. van Santen, J. A. Lercher, A. W. Kleyn, and H. J. Bakker, Chem. Phys. Lett. 278, 213 (1997).

${ }^{12}$ J. D. Beckerle, R. R. Cavanagh, M. P. Casassa, E. J. Heilweil, and J. C. Stephenson, J. Chem. Phys. 95, 5403 (1991).

${ }^{13}$ M. Morin, N. J. Levinos, and A. L. Harris, J. Chem. Phys. 96, 3950 (1992).

${ }^{14}$ H. Pfnür, D. Menzel, F. M. Hoffmann, A. Ortega, and A. M. Bradshaw, Surf. Sci. 93, 431 (1980).

${ }^{15}$ P. Jakob and B. N. J. Persson, Phys. Rev. B 56, 10644 (1997).

${ }^{16}$ T. Moritz and W. Widdra, Phys. Rev. Lett. 86, 103 (2001).

${ }^{17}$ M. Morin, N. J. Levinos, and A. L. Harris, J. Chem. Phys. 96, 3950 (1992).

${ }^{18}$ M. Morin, P. Jakob, N. J. Levinos, Y. J. Chabal, and A. L. Harris, J. Chem. Phys. 96, 6203 (1992).

${ }^{19}$ K. Kuhnke, M. Morin, N. J. Levinos, Y. J. Chabal, and A. L. Harris, J. Chem. Phys. 99, 6114 (1993).

${ }^{20}$ T. C. Shen, C. Wang, G. C. Abeln, J. R. Tucker, J. W. Lyding, Ph. Avouris, and R. Walkup, Science 268, 1950 (1995).

${ }^{21}$ Ch. Hess, M. Wolf, and M. Bonn, Phys. Rev. Lett. 85, 4341 (2000).
${ }^{22}$ Y. R. Shen, Nature (London) 337, 519 (1989), and references therein.

${ }^{23}$ E. W. M. van der Ham, Q. H. F. Vrehen, and E. R. Eliel, Opt. Lett. 21, 1448 (1996).

${ }^{24}$ L. J. Richter, T. P. Petralli-Mallow, and J. C. Stephenson, Opt. Lett. 23, 1594 (1998).

${ }^{25}$ M. Bonn, Ch. Hess, S. Funk, J. H. Miners, B. N. J. Persson, M. Wolf, and G. Ertl, Phys. Rev. Lett. 84, 4653 (2000).

${ }^{26}$ A. Tokmakoff, B. Sauter, A. S. Kwok, and M. D. Fayer, Chem. Phys. Lett. 221, 412 (1994).

${ }^{27}$ S. Funk, M. Bonn, D. N. Denzler, Ch. Hess, M. Wolf, and G. Ertl, J. Chem. Phys. 112, 9888 (2000).

${ }^{28}$ H. Pfnür, P. Feulner, and D. Menzel, J. Chem. Phys. 79, 4613 (1983).

${ }^{29} \mathrm{Ch}$. Hess, M. Bonn, S. Funk, and M. Wolf, Chem. Phys. Lett. 325, 139 (2000).

${ }^{30}$ J. H. Hunt, P. Guyot-Sionnest, and Y. R. Shen, Chem. Phys. Lett. 133, 189 (1987).

${ }^{31}$ A. L. Harris and L. Rothberg, J. Chem. Phys. 94, 2449 (1991).

${ }^{32}$ E. Knoesel, A. Hotzel, and M. Wolf, J. Electron Spectrosc. Relat. Phenom. 88-91, 577 (1998).

${ }^{33}$ R. W. Boyd, Nonlinear Optics (Academic, San Diego, 1992), p. 191.

${ }^{34}$ P. W. Atkins, Physical Chemistry, 4th ed. (Oxford University Press, Oxford, 1990).

${ }^{35}$ P. Jakob (private communication).

${ }^{36}$ P. Jakob and B. N. J. Persson, J. Chem. Phys. 109, 8641 (1998)

${ }^{37}$ J.-C. Diels and W. Rudolph, Ultrashort Laser Pulse Phenomena (Academic, San Diego, 1996).

${ }^{38}$ S. Andersson and B. N. J. Persson, Phys. Rev. Lett. 45, 1421 (1980).

${ }^{39}$ J. C. Kimball, C. Y. Fong, and Y. R. Shen, Phys. Rev. B 23, 4946 (1981).

${ }^{40}$ J. B. Eggert, H.-K. Mao, and R. J. Hemley, Phys. Rev. Lett. 70, 2301 (1993).

${ }^{41}$ P. Calvani, A. Nucara, M. C. Salvaggio, and S. Lupi, J. Chem. Phys. 101, 20 (1994).

${ }^{42}$ R. M. Shelby, C. B. Harris, and P. A. Cornelius, J. Chem. Phys. 70, 34 (1979).

${ }^{43}$ Note that the values for the hopping time reported here are a factor of 2 shorter than those reported in Ref. 21. This is due to the fact that in the previous calculations the transition dipole moment for the hot band $\left(\mu_{12}\right)$ was set equal to that of the fundamental $\left(\mu_{01}\right)$, which is incorrect, since $\mu_{12} \simeq 2 \times \mu_{01}$ (see text).

${ }^{44}$ Th. Förster, Discuss. Faraday Soc. 27, 7 (1959); Th. Förster, in Modern Quantum Chemistry, edited by O. Sininoğlu (Academic, New York, 1965), Part III, pp. 93-137.

${ }^{45}$ Laser Spectroscopy of Solids, 2nd ed., edited by W. M. Yen and P. M. Selzer (Springer-Verlag, Berlin, 1986).

${ }^{46}$ J. M. Drake, J. Klafter, and P. Levitz, Science 251, 1574 (1991), and references therein.

${ }^{47}$ A. M. van Oijen, M. Ketelaars, J. Köhler, T. J. Aartsma, and J. Schmidt, Science 285, 406 (1999).

${ }^{48}$ M. J. P. Brugmans, H. J. Bakker, and A. Lagendijk, J. Chem. Phys. 104, 64 (1996). 